\title{
OBSERVABILITY OF THE MAGNETIC FIELD IN MOLECULAR CLOUDS
}

\author{
N. BEL and B. LEROY \\ Observatoire de Paris-Meudon \\ 92195 Meudon Cedex \\ France
}

ABSTRACT. We have done detailed calculations of the Zeeman effect in the dozen diatomic molecules identified in interstellar clouds.

The magnetic field has become recognised as an unavoidable ingredient to be taken into account in the evolution of interstellar clouds.

The Zeeman splittings have been obtained from standard procedures of quantum mechanics. Only $\mathrm{CN}$ and so exhibit an effect comparable to that in $\mathrm{OH}$. The magnitude of this effect is best appreciated in terms of the frequency separation, $\Delta \nu(\mathrm{Hz})$, of the $\sigma$-components for a given magnetic field strength, B $(\mu \mathrm{G})$. For instance, for the transition NJF = $1,3 / 2,3 / 2 \rightarrow 0,1 / 2,1 / 2$ in $C N, \Delta \nu=2.2 \mathrm{~Hz} / \mu \mathrm{G}$, and for the transition $\mathrm{NJ}=2,1 \rightarrow 1,2$ in SO, $\Delta \nu=1.7 \mathrm{~Hz} / \mu \mathrm{G}$. (Recall that for the $1665 \mathrm{MHz}$ line of $\mathrm{OH}, \Delta \nu=3.27 \mathrm{~Hz} / \mu \mathrm{G}$.)

For the other observed diatomic molecules, Co, CS, SiO and SiS exhibit separations of the order of a few $10^{-4} \mathrm{~Hz} / \mu \mathrm{G}$, and the corresponding values for NO, NS and $\mathrm{CH}$ are still smaller.

To be detectable the signal should be greater than the minimum detectable temperature $\Delta T_{r m s}$ of the telescope which leads to a lower limit on the detectable magnetic field:

$$
B_{\text {min }} \approx \Delta T_{\text {rms }} \Delta \nu_{1} / \Delta \nu T_{A}^{*} \text {. }
$$

For the $30-\mathrm{m}$ antenna of IRAM

$$
\Delta \mathrm{T}_{\mathrm{rms}}=3-5 \mathrm{mK}
$$

for a system temperature $\mathrm{T}_{\text {sys }}=600$ or $1000 \mathrm{~K}$, according to whether the 230 or $115 \mathrm{GHz}$-receptor is used, a typical pre-detection bandwidth of $1 \mathrm{MHz}$ and an observing time $\tau \approx 50 \mathrm{~h}$ (which is of the order of the observation time for $\mathrm{OH}$ observations); then $\mathrm{B}_{\mathrm{min}}$ reaches a few $100 \mu \mathrm{G}$.

This value is of the order of magnitude one could expect in moderately dense clouds, and well below the magnetic strength inferred near the Galactic Centre.

Let us note that the molecule $\mathrm{O}_{2}$ exhibits a pronounced Zeeman effect, even stronger than that of $\mathrm{OH}$. Unfortunately, these transitions are strongly absorbed in the atmosphere. 